\title{
Electronic phases of substances. Phase transitions with change of electron and crystalline structure
}

\author{
Boris Nadykto ${ }^{\mathrm{a}}$ \\ Russian Federal Nuclear Center - VNIIEF, Sarov, Nizhni Novgorod region, 607190, Russia
}

\begin{abstract}
There is plenty of experimental data on high-pressure phase transformations in various materials. Variations in materials characteristics (for example, equilibrium density and bulk modulus), while the crystalline structure remains unchanged, are indicative of energy variations in outer-shell electrons of solid atoms. In experiments with crystalline structure variations, the dependence of pressure on density in some cases can be described by the same curve, the parameters of which are independent of the crystalline structure. Examples of such transformations in some materials at static compression and in shock-wave experiments are given.
\end{abstract}

\section{Introduction}

In the last decade, numerous publications have appeared describing complex variations in the crystalline structure of "simple" alkaline and alkaline-earth elements under compression [1-4]. These variations drive changes in many characteristics, in particular, electrical conductivity. This paper will focus on the variations in the $\mathrm{P}(\rho)$ relationship, and the behavior of such elements in this respect is similar to the behavior of many other elements. Shock and static compression experiments reveal highpressure variations in the structure of materials that manifest themselves as kinks or even discontinuities in the $\mathrm{P}(\rho)$ curve. In some cases, when the crystalline structure of the material is unchanged, such kinks can be attributed only to a change in the electronic structure of solid atoms (i.e. redistribution of electrons between inner and outer atomic shells).

The notion of solid electronic phases appeared in the late 1940s, when it was detected from X-ray diffraction measurements of samples under pressure [5] that different cerium phases had the same type of crystalline lattice, but different density. Fermi suggested that different phase characteristics were due to the different electronic structure of solid atoms in different phases. During a phase transition, electrons are redistributed between atomic shells, while the crystalline structure is preserved. Based on Fermi's concept, Steinheimer in [6] calculated characteristics of two isostructural phases of cesium.

\section{Computational analysis of the structure of solids}

Nadykto [7-9] suggested a method for calculating the energy of a compressed atom. For the case of confined volume per atom (i.e. for an atom within a solid), a system of energy levels distinct from those of a

\footnotetext{
${ }^{a}$ Corresponding author: nadykto@vniief .ru
}

free atom was obtained. The model explicitly includes the quantum nature of electron motion in solid atoms. Application of this model to a unit cell of a solid atomic lattice leads to a semiempirical equation of state that relates material compressibility to compression of the outer electron shell of an atom within a solid. Solids display a peculiar effect of both quantum properties of the system (discrete angular momentum of electrons and related energy discontinuity) and continuous energy and pressure variation with a change in external conditions (for example, during compression), where the quantum state remains unchanged.

Elastic energy of a solid is a sum of the kinetic energy of electrons provided that their angular momentum is quantized and potential energy of their interaction with the nucleus charge and each other. Different materials and their different phases are described by the same analytical expression for the equation of state. Only parameters of the equation of state differ: equilibrium density and bulk modulus (at $\mathrm{P}=0, \mathrm{~T}=0$ ) or energy of outer-shell electrons of the atomic cell of a solid.

Energy and pressure are written as a sum of cold (elastic, potential) and thermal components:

$$
\begin{aligned}
& E=E_{X}+E_{T} \\
& P=P_{X}+P_{T} .
\end{aligned}
$$

The elastic components of energy and pressure are defined as

$$
\begin{gathered}
E(\sigma)=\frac{9 B_{0}}{\rho_{n}}\left(\frac{\sigma^{2 / 3}}{2}-\sigma^{1 / 3}\right) \\
P(\sigma)=3 B_{0}\left(\sigma^{5 / 3}-\sigma^{4 / 3}\right)
\end{gathered}
$$

where $\sigma=\rho / \rho_{n}$ is the compression ratio, and $\rho_{n}$ is the equilibrium material density at $P=0, T=0$. 
Table 1. Equation-of-state parameters for some elements.

\begin{tabular}{|l|l|l|l|}
\hline Element & $\rho_{n}, \mathrm{~g} / \mathrm{cm}^{3}$ & $\mathrm{~B}_{0}, \mathrm{GPa}$ & $\mathrm{E}_{n}, \mathrm{eV}$ \\
\hline $\mathrm{Li}$ & 0.548 & 13.7 & 8.07 \\
\hline $\mathrm{Li}$ & 0.415 & 6.5 & 3.84 \\
\hline $\mathrm{Na}$ & 1.00 & 7.8 & 8.4 \\
\hline $\mathrm{Na}$ & 1.18 & 15.7 & 14.3 \\
\hline $\mathrm{K}$ & 0.892 & 4,0 & 8.11 \\
\hline $\mathrm{Rb}$ & 1.616 & 3.1 & 7.7 \\
\hline $\mathrm{Rb}$ & 2.02 & 4 & 7.9 \\
\hline $\mathrm{Zn}$ & 7.21 & 62.7 & 26.5 \\
\hline $\mathrm{Zn}$ & 7.5 & 114 & 46.5 \\
\hline $\mathrm{Zn}$ & 9.18 & 337 & 112 \\
\hline $\mathrm{Th}$ & 11.78 & 50 & 50.8 \\
\hline $\mathrm{Th}$ & 12.31 & 76 & 66.8 \\
\hline $\mathrm{Th}$ & 18.63 & 360 & 209 \\
\hline $\mathrm{Pa}$ & 15.37 & 115 & 80.6 \\
\hline $\mathrm{Pa}$ & 19.83 & 400 & 217 \\
\hline $\mathrm{Am}$ & 13.67 & 28.5 & 23.4 \\
\hline $\mathrm{Am}$ & 11.89 & 13 & 12.3 \\
\hline $\mathrm{Am}$ & 18.59 & 80 & 48.4 \\
\hline $\mathrm{Am}$ & 24.46 & 350 & 161 \\
\hline
\end{tabular}

The Mie-Grueneisen form of the thermal component of pressure is $P_{T}=\Gamma \rho E_{T}$. The Grueneisen coefficient $\Gamma$ is computationally estimated using relations (3), (4) in the Dougdale-Macdonald approximation.

With a known bulk modulus $\mathrm{B}_{0}$, it is possible to determine the effective energy of state of outer-shell electrons in the atomic cell of a solid:

$$
E_{n}=\frac{9 A B_{0}}{2 N_{A} \rho_{n}} .
$$

Varying the number and quantum state of outershell electrons results in a non-monotone behavior of compressibility (electronic phase transition), which is observed when comparing simulations with experimental data.

Table 1 shows the equation-of-state parameters thus obtained for the elements discussed below.

\subsection{Alkaline metals}

\subsubsection{Lithium}

At low temperatures $(200 \mathrm{~K})$, lithium undergoes a series of transformations at pressures up to $130 \mathrm{GPa}$ : bcc fcc - cI16 - oC88 - oC40 - oC24 [4]. However, at pressures up to $80-100 \mathrm{GPa}$, the $\mathrm{P}(\rho)$ diagram of statically compressed lithium can be described by common parameters of equation of state (4) that do not depend on the crystalline structure. The complex behavior of lithium is observed under shock compression [10], [11]. The experiments were performed in a limited range of pressures. The results of measurements in [10] taken at pressures up to $25 \mathrm{GPa}$ closely match the equation of state describing the experimental isotherm from [4]. However, at higher pressures $(30-70 \mathrm{GPa})$ the data from [11] on the Hugoniot deviate significantly from the computational curve obtained using the initial elastic compression curve. The description at once of the Hugoniot and the isotherm (or cold curve) is indicative of a potential change in the

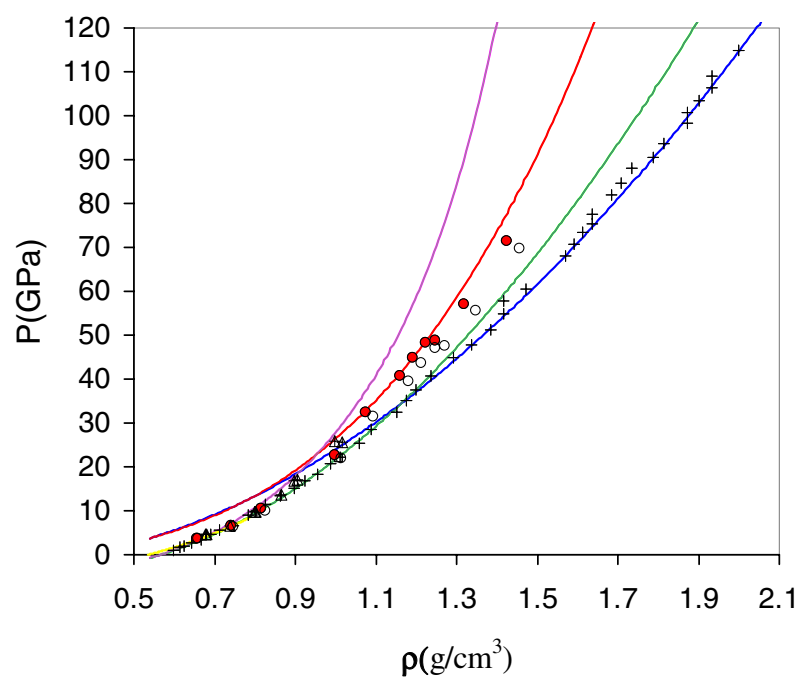

Figure 1. Isotherm at $190 \mathrm{~K}$ [4] and Hugoniot [10,11] of Li. The curves represent calculations, and the points show experimental values.

electronic structure of compressed Li. The data in [11] point at a change in the slope of the D-u curve. However, in the range, where the data from [10] and [11] overlap, the data from [11] lie $300 \mathrm{~m}$ below [10] along D.

If this is associated with a systematic error in [11], then at high pressure the values of $\mathrm{P}(\rho)$ from [11] shift a little bit up. Fig. 1 shows our computational curves along with the experimental data of [10], [11] (Hugoniot) and [4] (Diamond anvil sell), and well as corrected data from [11]. Above the $1.2 \mathrm{~g} / \mathrm{cm}^{3}$ density, the data on the isotherm and the corrected data from [11] are described by the same equation-of-state parameters, which differ from the parent phase parameters. The new parameters point at a transition of electrons from the $1 s^{2} 2 s$ state to $1 s^{2} 2 p$ in compressed lithium. To render conclusions more definite, new shock-wave experiments on Li need to be conducted at pressures of 30-100 GPa. The calculated parameters of different phases of Li are given in Table 1.

\subsubsection{Sodium}

The experimental data on static compression of $\mathrm{Na}[4,12]$ up to $160 \mathrm{GPa}$ (compression ratio 5) can be described by two curves. The parent phase parameters describe the experimental data up to $\mathrm{P}=7.5 \mathrm{GPa}$. Note that at 7.5 GPa the crystalline structure of $\mathrm{Na}$ remains unchanged. At $\mathrm{P}=7.5-160 \mathrm{GPa}$, experimental points are accurately described by the high-pressure phase parameters (see Table 1). Figure 2 shows a comparison of calculated and experimental data.

The bcc-to-fcc transition in Na observed experimentally at $65 \mathrm{GPa}$ does not essentially affect the $\mathrm{P}(\rho)$ curve. The presented parameters of the electronic phases of $\mathrm{Na}$ accurately describe the data of shock experiments [10], [11], which closely match each other. The following series of transformations is observed in [4] up to $160 \mathrm{GPa}$ : bcc fcc $-\mathrm{cI} 16-\mathrm{oP} 8-\mathrm{tI} 19$. All these phases at $7.5-180 \mathrm{GPa}$ are described by the same equation-of-state parameters, which differ from those of the parent phase. 


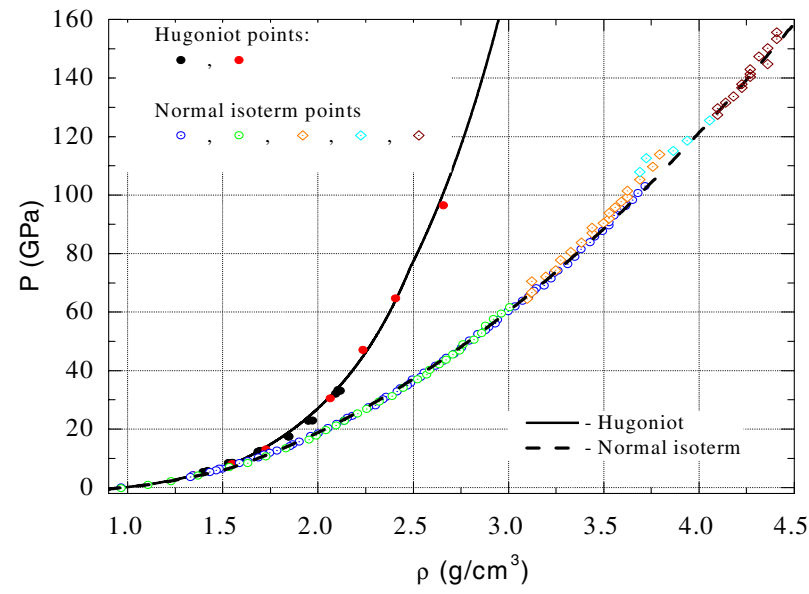

Figure 2. Isotherm at $300 \mathrm{~K}[4,12]$ and Hugoniot [10,11] of $\mathrm{Na}$. The curves represent calculations, and the points show experimental values.

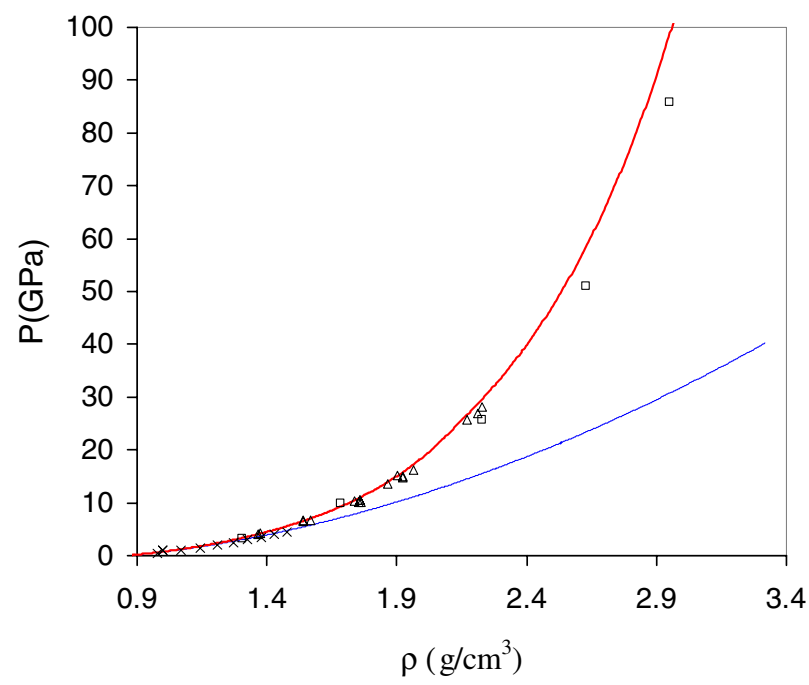

Figure 3. Isotherm at $300 \mathrm{~K}$ [4] and Hugoniot [10,11] of potassium. The curves represent calculations, and the points show experimental values.

\subsubsection{Potassium}

The experimental data on compression of potassium are rather scarce. Reference [13] shows the results of processing the data of static-compression experiments conducted by P. Bridgeman at pressures up to $4.5 \mathrm{GPa}$. Simulations with parent-phase equation-of-state parameters provide a satisfactory description of these data and results of shock experiments [10,11]. Figure 3 shows a comparison of calculated and experimental data. No phase transformation discontinuities are observed in the $\mathrm{P}(\rho)$ curve up $85 \mathrm{GPa}$.

\subsubsection{Rubidium}

For rubidium, in addition to the Hugoniot from [10] and static-compression data [13], there are diamondanvil measurement results [14], which clearly point at a phase transition at 13-18 GPa. Figure 4 shows calculated and experimental data. As one can see in Table 1, the energy of atoms in these phases of $\mathrm{Rb}$ is virtually the same. Therefore, one can suppose that the transition

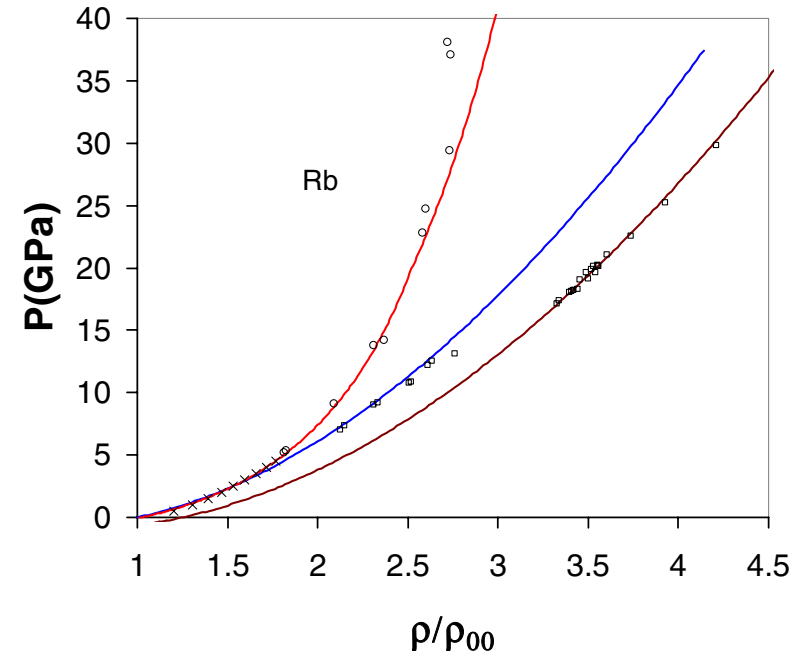

Figure 4. Isotherm at $300 \mathrm{~K}[13,14]$ and Hugoniot $[10,11]$ of rubidium. The curves represent calculations, and the points show experimental values.

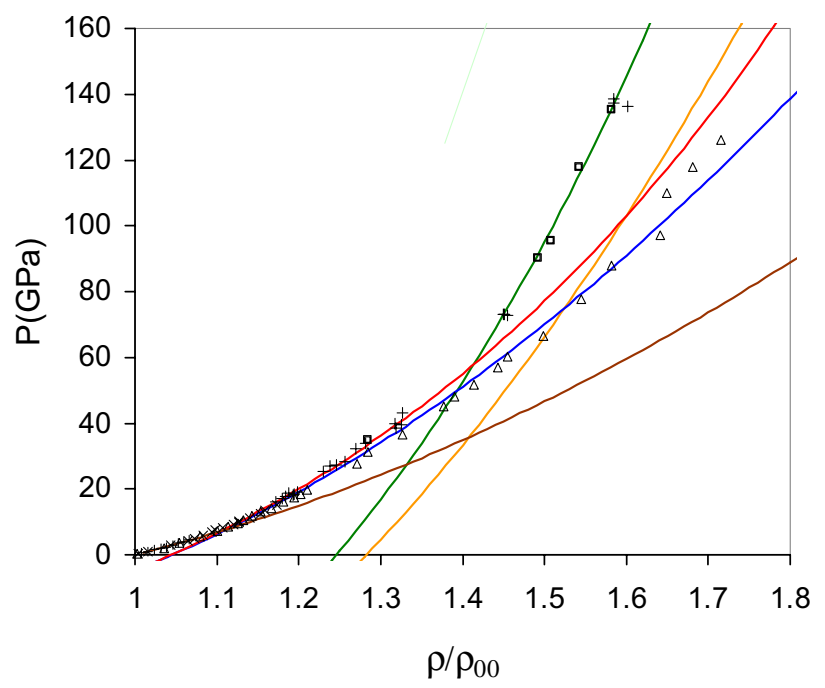

Figure 5. Pressure $P(\rho)$ on the normal isotherm and Hugoniot in zinc. The points represent experimental data. The curves represent calculations for different electronic phases of zinc.

occurs, while the electronic structure of $\mathrm{Rb}$ atoms remains unchanged.

\subsection{Zinc}

The equation-of-state parameters for zinc obtained by processing the data of shock-wave experiments [10], [15] differ from the parent-phase parameters of zinc. Description of the experimental points on the Hugoniot suggests that there are phase transitions at $7.5 \mathrm{GPa}$ and $57 \mathrm{GPa}$. The parent- and the high-pressure-phase parameters of zinc are given in Table 1. Calculated Hugoniots and experimental points are shown in Fig. 5. These parameters refer to the equilibrium state at $P=0$, $T=0$. Considering thermal expansion, the density of zinc under normal conditions is $\rho_{00}=7.14 \mathrm{~g} / \mathrm{cm}^{3}$ and the bulk modulus is $B_{0}=61.0 \mathrm{GPa}$. 
Figure 5 also shows experimental points on the normal isotherm of zinc from $[13,16]$. Calculations for the parent phase are in perfect agreement with [13] up to 4.5 GPa. Data on static compression of zinc in [15] coincide with the computational curve for the parent phase at low pressures and with the computational curve for the high-pressure phase at $P>7.5 \mathrm{GPa}$.

The data in [17] suggest that the hcp lattice of zinc is stable up to $126 \mathrm{GPa}$. Hence, one can say that the observed phase transitions in zinc are electronic phase transitions with unchanged crystalline structure. The parent phase atomic cell energy $26.5 \mathrm{eV}$ in zinc is close to the energy of two outer-shell electrons of the free atom of zinc in the $3 d^{10} 4 s^{2}$ state. The energy of three outer-shell electrons of the free atom of zinc is $67 \mathrm{eV}$ and that of four electrons is $129 \mathrm{eV}$.

The electronic phase transition with material crystalline structure remaining unchanged was experimentally detected in zinc [16] from the measured ratio of axes $c / a$ of the hexagonal close-packed structure of zinc as a function of $V / V_{0}$, which showed a non-monotonicity at $9 \mathrm{GPa}$. Existence of the electronic phase transition in zinc at $P=6.6 \mathrm{GPa}$ and $T=4.2 \mathrm{~K}$ also follows from Moessbauer spectroscopy [18], which suggests a drastic change in the crystalline lattice dynamics in this transition. In [16], [18], this transition is treated as a Lifshitz topological phase transition (TPT) [19]. Since 1995, there have been published a lot of experimental and theoretical investigations to prove the existence of TPT in zinc, which yielded contradictory results (see, e.g., [20]). Our analysis of zinc compressibility clearly points to this electronic phase transition in zinc at $P=7.5 \mathrm{GPa}$, as there is a kink in the slope of the $\mathrm{P}(\rho)$ curve (jump in the bulk compression modulus). In addition, the analysis infers the existence of another electronic phase transition at $P=57 \mathrm{GPa}$, with the crystalline structure remaining unchanged, too. The electronic phase transitions similar to those in zinc can be observed in $\mathrm{Cd}, \mathrm{Tl}, \mathrm{Pd}, \mathrm{Ag}, \mathrm{Sb}$, and other elements.

\subsection{Actinides}

\subsubsection{Thorium}

In [21], the $\mathrm{P}(\rho)$ diagram on the normal isotherm in thorium was measured up to a pressure of $300 \mathrm{GPa}$. At pressures above $25 \mathrm{GPa}$, thorium displays high-pressure phases, first with $69.5 \mathrm{eV}$ energy corresponding to four electrons in the outer shell, and then there is a transition to a phase of $19.1 \mathrm{~g} / \mathrm{cm}^{3}$ equilibrium density, $400 \mathrm{GPa}$ bulk modulus, and $227 \mathrm{eV}$ outer-shell electron energy per atom. This energy corresponds to six electrons in the outer shell, and this means that the electron rearrangement involves completed shells.

In the presence of phase transitions, different phase states of the same density can be observed in static and shock-compression experiments. Thus, the kink in the $\mathrm{P}(\rho)$ curve on the normal isotherm of thorium [21] that can be attributed to a change in the quantum state of outershell electrons occurs at $\mathrm{P}=150 \mathrm{GPa}$ and compression ratio $\sigma=2.2$. The kink in the Hugoniot of thorium at $\mathrm{P}=80 \mathrm{GPa}$ is adequately described by parameters of the

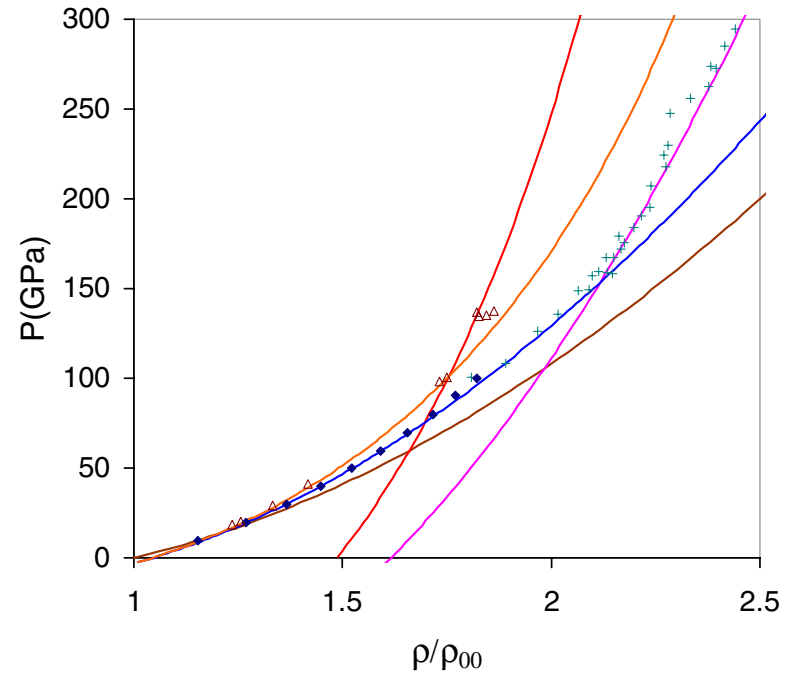

Figure 6. Isotherm at $300 \mathrm{~K}$ [21] and Hugoniot [10] of thorium. The curves represent calculations, and the points show experimental values.

high-pressure phase appearing on the normal isotherm at $\mathrm{P}=150 \mathrm{GPa}$. This means that in the compression range of $\sigma=1.7-2.2$ the elastic component of pressure on the Hugoniot in thorium differs from the values on the normal isotherm (see Fig. 6).

\subsubsection{Protoactinium}

In [22], compressibility of protoactinium was studied up to a pressure of $129 \mathrm{GPa}$ by the diamond-anvil-cell method and X-ray diffraction with a synchrotron source of radiation. The computational analysis indicates that the experimental points lie on the calculated curve with $\rho_{0}=$ $15.37 \mathrm{~g} / \mathrm{cm}^{3}$ and $\mathrm{B}_{0}=115 \mathrm{GPa}$ up to $\mathrm{P} \approx 95 \mathrm{GPa}$. At higher pressures, the experimental points deviate significantly from the calculated curve and can be described as states of a different electronic phase of protactinium with parameters from Table 1. Figure 7 shows calculated curves and experimental points on the normal isotherm of protactinium from [22].

The authors of [22] note that the tetragonal structure of protactinium transfers to the low-symmetry orthorhombic structure of $\alpha$-uranium at $\mathrm{P}=77 \mathrm{GPa}$. As one can see in Fig. 7, there is no noticeable change in the slope of the $\mathrm{P}(\rho)$ curve at $77 \mathrm{Gpa}$, which is indicative of the unchanged initial electron structure of protactinium. An abrupt change in the slope and electronic structure occurs at $94 \mathrm{GPa}$, apparently, with the unchanged orthorhombic crystalline structure of $\alpha$-uranium.

\subsubsection{Americium}

In [23], Lindbaum et al. obtained high-pressure experimental data for americium up to $\mathrm{P}=100 \mathrm{GPa}$. The transition from the AmI phase to the AmII and AmIII phases leads to a change in the slope of the $\mathrm{P}(\rho)$ curve that corresponds to a drop in the bulk modulus (more than by a factor 


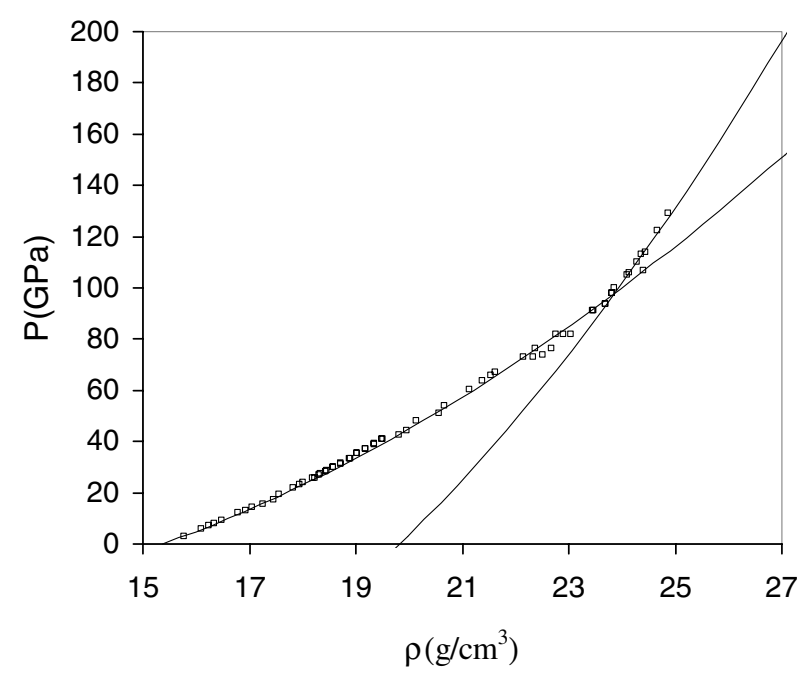

Figure 7. Isotherm at $300 \mathrm{~K}$ [22] for protactinium. The curves represent calculations, and the points show experimental values.

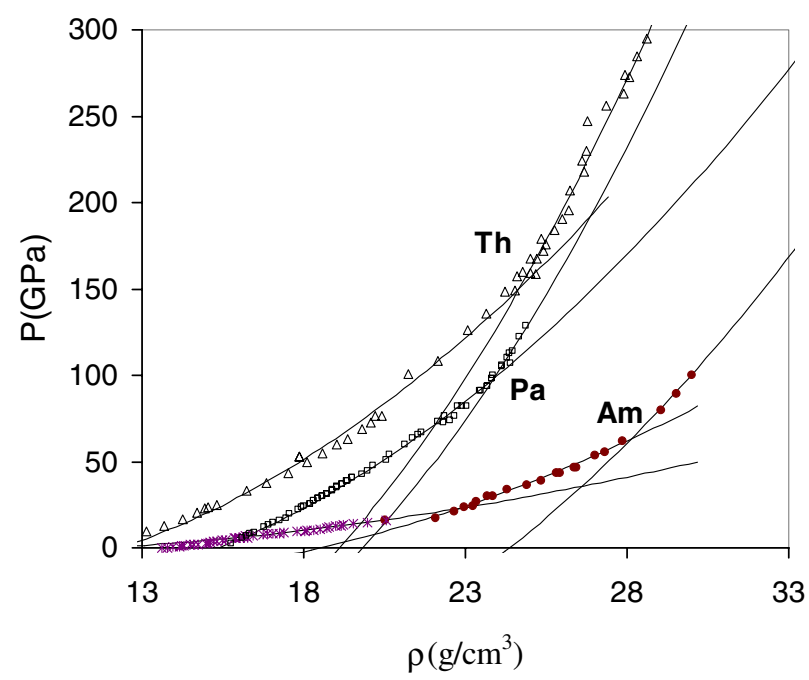

Figure 8. Isotherm at $300 \mathrm{~K}$ [23] for Th, Pa, U, Am. The curves represent calculations, and the points show experimental values.

of 2) and increase in the equilibrium volume for these phases. Figure 8 shows calculated curves and experimental points on the normal isotherm of americium from [23]. Despite the different crystalline structure of the AmII and AmIII phases, their $\mathrm{P}(\rho)$ diagram can be described by a single curve with the same values of bulk modulus, $\mathrm{B}_{0}=$ 13.0 GPa, and equilibrium density, $\rho_{n}=11.9 \mathrm{~g} / \mathrm{cm}^{3}$.

In americium, an abrupt increase in the bulk modulus occurs with a transition to the AmIV phase. Most of experimental points for the phase lie on the calculated curve with $\mathrm{B}_{0}=80 \mathrm{GPa}$ and $\rho_{n}=18.6 \mathrm{~g} / \mathrm{cm}^{3}$. However, three upper experimental points dramatically deviate from the calculated curve and are described by $\mathrm{B}_{0}=350 \mathrm{GPa}$ and $\rho_{n}=24.5 \mathrm{~g} / \mathrm{cm}^{3}$. This change suggests a significant rearrangement of the electronic structure of the outer electron shell under pressure.

\section{Conclusion}

The analysis shows that many materials exhibit peculiar properties under compression, which is due to different states of atomic cell electrons in solids. The variety of electronic phases in solids is a reflection of the diversity of energies of excited electronic states observed in atomic spectroscopy.

Simple alkaline metals, just as transition metals and actinides, are characterized by a complex behavior under compression, with changes both in the crystalline and in the electronic structure.

In the experiments with crystalline structure variation, the pressure-density relationship can be described by the same curve, the parameters of which are independent of the crystalline structure.

\section{References}

[1] E.G. Maksimov, M.V. Magnitskaya, V.E. Fortov, Uspekhi Fiz. Nauk 175, 793 (2005)

[2] E. Gregoryanz, O. Degtyareva, M. Somayazulu et al. Physical Review Letters 94, 185502 (2005)

[3] E. Gregoryanz, L.F. Lundegaard, M.I. McMahon et al. Science 320(5879), 1054 (2008)

[4] C.L. Guillaume, E. Gregoryanz, O. Degtyareva et al. Nature Physics Letters, 7(3), 211 (2011)

[5] A.W. Lawson, T.Y. Tang, Phys. Rev. 76, 301 (1949)

[6] R. Steinheimer, Phys. Rev. 78, 235 (1950)

[7] B.A. Nadykto, Uspekhi Fiz. Nauk 163, 37 (1993)

[8] B.A. Nadykto, Vopr. Atomnoi Nauki i Tekhniki, Sr. Teor. I Prikl. Fizika 3, 58 (1996)

[9] B.A. Nadykto, Rusian Chemical Journal XLV (5-6), 7 (2001)

[10] LASL Shock Hugoniot Data. Ed. Marsh S.P. Berkeley - Los Angeles - London: University of California Press, 1980. 658.

[11] A.A. Bakanova, I.P. Dudoladov, R.F. Trunin, Fizika Tverdogo Tela 7, 1615 (1965)

[12] M. Hanfland, I. Loa, K. Syassen, Phys. Rev. B 65, 184109 (2002)

[13] S.N. Vaidya, G.C. Kennedy, Phys. Chem. Solids 31, 2329 (1970)

[14] U. Schwarz, A. Grechnik, K. Syassen et al., Physical Review Letters 83, 4085 (1999)

[15] L.V. Altshuler, A.A. Bakanova, R.F. Trunin, ZheTF 42, 91 (1962)

[16] K. Takemura, Phys. Rev. Lett. 75, 1807 (1995)

[17] K. Takemura, Phys. Rev., B 56, 5170 (1997)

[18] Potzel W., Steiner M., Karzel H et al., Phys. Rev. Lett. 74, 1139 (1995)

[19] I.M. Lifshitz, Zh. Eksp. Theor. Fiz. 38, 1569 (1960) [Sov. Phys. JETP, 11, 1130 (1960)]

[20] B.K. Godval, P. Modak, R.S. Rao, Bull. Mater. Sci. 26, 175 (2003)

[21] Y.K. Vohra, J. Akella, Phys. Rev. Lett. 67, 3563 (1991)

[22] R.G. Haire, S. Heathman, M. Idiri et al. Phys. Rev., 2003, B 67, 134101

[23] A. Lindbaum, S. Heathman, K. Litfin. et al., Phys. Rev. B 63, 214101 (2001) 